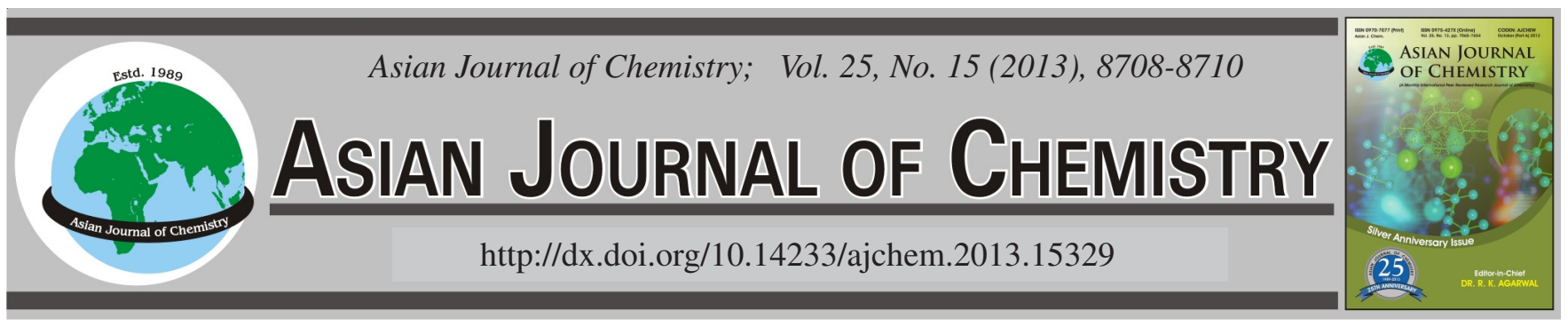

\title{
Microwave-Assistant Synthesis, Crystal Structure and Fungicidal Activity of 3-Chloro-2-hydrazinylpyridine
}

\author{
WeI KE ${ }^{1}, \mathrm{NA}_{\mathrm{B}} \mathrm{Bo} \mathrm{SuN}^{1}$ and Hong-Ke Wu${ }^{2, *}$
}

${ }^{1}$ College of Biology and Environmental Engineering, Zhejiang Shuren University, Hangzhou 310015, Zhejiang Pronvince, P.R. China ${ }^{2}$ College of Chemical Engineering and Materials Science, Zhejiang University of Technology, Hangzhou 310014, Zhejiang Province, P.R. China

*Corresponding author: E-mail: wuhk910@gmail.com

\begin{abstract}
A pyridine derivative i.e., 3-chloro-2-hydrazinylpyridine $\left(\mathrm{C}_{5} \mathrm{H}_{6} \mathrm{~N}_{3} \mathrm{Cl}\right)$ was synthesized under microwave irradiation and its structure was studied by X-ray diffraction and ${ }^{1} \mathrm{H}$ NMR. The crystals are monoclinic, space group $\mathrm{p} 2{ }_{1} / \mathrm{c}$ with $\mathrm{a}=11.6276(14), \mathrm{b}=3.8924(5), \mathrm{c}=$ 13.9558 (17) $\AA, \alpha=90.00, \beta=103.447(6), \gamma=90.00^{\circ}, V=614.31(13) \AA^{3}, Z=4, F_{(000)}=296, D_{c}=1.552 \mathrm{~g} / \mathrm{cm}^{3}, \mu=0.52 \mathrm{~cm}^{-1}$, the final $R$ $=0.0623$ and $w R=0.1897$. A total of 7008 reflections were collected, of which 1406 were independent $\left(R_{\text {int }}=0.0544\right)$. The fungicidal activity of this compound was also studied.
\end{abstract}

Key Words: Microwave assistant synthesis, Crystal structure, Biological activity.

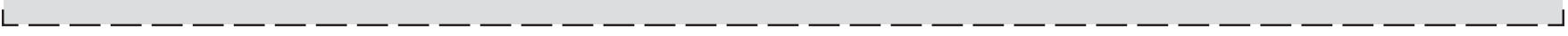

\section{INTRODUCTION}

In recent years, heterocyclic compounds had received considerable attentions because of their important biological activity $^{1}$. So the synthesis of broader spectrum and highly bioactive compounds becomes the mainstream in the medicinal and agriculture chemistry field ${ }^{2}$. Pyridine derivatives also exhibited excellent property, such as nicotinate mononucleotide adenylyltransferase inhibitor ${ }^{3}$, antifungal activity $^{4}$, antiinflammatory activity ${ }^{5}$, antimicrobial activities ${ }^{6}$, anticancer activity ${ }^{7}$, antiviral activity $^{8}$, cholesterol absorption inhibitors 9 . Microwave technique has been widely used for a variety of organic reactions, such as Claisen, heterocyclic synthesis, oxidation, hydrolysis, esterification, etherification, etc.

In order to search for new compounds with good biological activity, a pyridine compound was synthesized under microwave irradiation. Their structures are confirmed by ${ }^{1} \mathrm{H}$ NMR and single crystal. The preliminary biological tests show that these compounds had moderate fungicidal activity.

\section{EXPERIMENTAL}

Melting points determined by a Yanaco MP-241 apparatus and uncorrected. Infrared spectra were recorded on a Bruker Equinox 55 spectrophotometer as $\mathrm{KBr}$ tablets. ${ }^{1} \mathrm{H} \mathrm{NMR}$ spectra were measured on a Bruker AC-P500 instrument (300 MHz) using TMS as internal standard and $\mathrm{CDCl}_{3}$ as solvent. Crystallographic data of the compound collected on a BRUCKER CCD SMART diffractometer. All chemicals were of AR grade.
Crystal structure determination: The crystal of 3-chloro2-hydrazinylpyridine with dimensions of $0.12 \mathrm{~mm} \times 0.08$ $\mathrm{mm} \times 0.06 \mathrm{~mm}$ was mounted on a Rigaku Saturn CCD areadetector diffractometer with a graphite-monochromated $\mathrm{MoK}_{\alpha}$ radiation $(\lambda=0.71073 \AA$ ) by using a phi and scan modes at 294(2) $\mathrm{K}$ in the range of $3.1^{\circ} \leq \theta \leq 27.7^{\circ}$. The crystal belongs to monoclinic system with space group $\mathrm{P} 2{ }_{1} / \mathrm{C}$ and crystal parameters of $\mathrm{a}=11.6276(14) \AA, \mathrm{b}=3.8924(5) \AA, \mathrm{c}=$ $13.9558(17) \AA, \alpha=90^{\circ} \beta=103.447(6)^{\circ}, \gamma=90^{\circ}, \mathrm{V}=$ 614.31(13) $\mathrm{A}^{3} \mathrm{D}_{\mathrm{c}}=1.552 \mathrm{~g} / \mathrm{cm}^{3}$. The absorption coefficient $\mu$ $=0.520 \mathrm{~mm}^{-1}$ and $\mathrm{Z}=4$. The structure was solved by direct methods with SHELXS-97 and refined by the full-matrix least squares method on $\mathrm{F}^{2}$ data using SHELXL-97. The empirical absorption corrections were applied to all intensity data. $\mathrm{H}$ atom of N-H was initially located in a difference Fourier map and were refined with the restraint $\operatorname{Uiso}(\mathrm{H})=1.2 \mathrm{Ueq}(\mathrm{N})$. Other $\mathrm{H}$ atoms were positioned geometrically and refined using a riding model, with $\mathrm{d}(\mathrm{C}-\mathrm{H})=0.93-0.97 \AA$ and $\operatorname{Uiso}(\mathrm{H})=1.2$ $\mathrm{Ueq}(\mathrm{C})$ or $1.5 \mathrm{Ueq}(\mathrm{Cmethyl})$. The final full-matrix least squares refinement gave $\mathrm{R}=0.0623$ and $\mathrm{wR}=0.1897$.

Synthesis: A modified two-phase procedure was applied. 2,3-Dichloropyridine $(0.2 \mathrm{~mol})$, hydrazine hydrate $(85 \%, 1$ mol) were put in a sealed vial, then refluxed at $100^{\circ} \mathrm{C}$ for 0.5 $\mathrm{h}$ under microwave irradiation. The product was obtained after filtrated. ${ }^{1} \mathrm{H}$ NMR $\left(\mathrm{CDCl}_{3}, 400 \mathrm{MHz}\right): 3.97$ (br. s, $\left.2 \mathrm{H}, \mathrm{NH}_{2}\right)$, 6.21 (br. s, 1H, NH), 6.64 (m, 1H, pyridyl-H), 7.47 (d, 1H, J= $7.6 \mathrm{~Hz}$, pyridyl-H), 8.09 (d, 1H, $J=4.9 \mathrm{~Hz}$, pyridyl-H). 


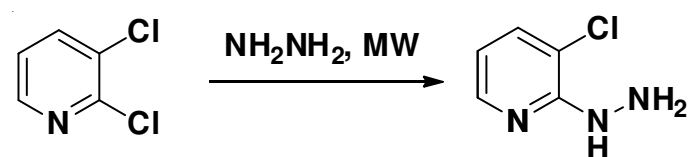

Scheme-I: Synthesis route of 3-chloro-2-hydrazinylpyridine

\section{RESULTS AND DISCUSSION}

Synthesis and spectroscopic properties: The title compound was synthesized under conventional and microwave irradiation condition. If the title compound was synthesized under refluxing, it should react with ethanolic solution of $\mathrm{NH}_{2} \mathrm{NH}_{2}$ for $72 \mathrm{~h}$. Surprisingly, it was synthesized for $0.5 \mathrm{~h}$ under microwave irradiation. ${ }^{1} \mathrm{H}$ NMR spectrum of the title compound tested shows 3.97 and 6.21 is the peak of $\mathrm{NH}_{2}$ and $\mathrm{NH}$, respectively. The 6.647.478.09 can be assigned to the three $\mathrm{CH}$ of pyridine. The melting point is according to the reference.

Structure of the title compound: Crystallographic and refinement parameters are given in Table- 1 . The selected bond lengths and bond angles listed in Tables 2-4, respectively. The structure was solved by direct methods. Anisotropic displacement parameters were applied to all nonhydrogen atoms in full-matrix least-square refinements based on $\mathrm{F}^{2}$. The hydrogen atoms were set in calculated positions with a common fixed isotropic thermal parameter.

\begin{tabular}{|c|c|}
\hline \multicolumn{2}{|c|}{$\begin{array}{c}\text { TABLE-1 } \\
\text { CRYSTAL DATA AND STRUCTURE } \\
\text { REFINEMENT FOR THE TITLE COMPOUND }\end{array}$} \\
\hline Items & Values \\
\hline Empirical formula & $\mathrm{C}_{5} \mathrm{H}_{6} \mathrm{~N}_{3} \mathrm{Cl}$ \\
\hline Formula weight & 143.58 \\
\hline Crystal system & monoclinic \\
\hline Space group & $\mathrm{p} 2_{1} / \mathrm{c}$ \\
\hline Unit cell & mensions \\
\hline $\mathrm{a}(\AA)$ & $11.6276(14)$ \\
\hline $\mathrm{b}(\AA)$ & $3.8924(5)$ \\
\hline c $(\AA)$ & $13.9558(17)$ \\
\hline \multicolumn{2}{|c|}{ Unit cell angles $\left({ }^{\circ}\right)$} \\
\hline$\alpha$ & 90 \\
\hline$\beta$ & $103.447(6)$ \\
\hline$\gamma$ & 90 \\
\hline Volume $\left(\AA^{3}\right)$ & $614.31(13)$ \\
\hline $\mathrm{Z}$ & 4 \\
\hline Temperature (K) & $296(2)$ \\
\hline Wavelength $(\AA)$ & 0.71073 \\
\hline Calculated density $\left(\mathrm{g} / \mathrm{cm}^{3}\right)$ & 1.552 \\
\hline Absorption coefficient $\left(\mathrm{mm}^{-1}\right)$ & 0.520 \\
\hline$F_{(000)}$ & 296 \\
\hline Theta range for data collection $\left({ }^{\circ}\right)$ & $1.80-27.72$ \\
\hline Reflections collected & 7008 \\
\hline Independent reflections & $1406\left[\mathrm{R}_{\text {(int) }}=0.0544\right]$ \\
\hline Final $R$ indices $[\mathrm{I}>2 \sigma(\mathrm{I})]$ & $\mathrm{R}_{1}=0.0623, \mathrm{wR}_{2}=0.1897$ \\
\hline
\end{tabular}

The molecular structure and atom labels are shown in Fig. 1. The one-dimensional linework of hydrogen bonds (dashed lines) is illustrated in Fig. 2, respectively.

In Table-3, the results indicate that the lengths of three $\mathrm{C}$ $\mathrm{N}$ bond C5-N3, C1-N2 and N3-C1 are 1.348(5), 1.365(4) and $1.335(4) \AA$, respectively, which are all longer than those in the single heterocycle ring. However, the C5-N3, C1-N2
TABLE-2

SELECTED BOND LENGTHS [A] FOR THE TITLE COMPOUND

\begin{tabular}{cccc}
\hline Bond lengths & X-Ray crystal & Bond lengths & X-Ray crystal \\
\hline $\mathrm{Cl}(1)-\mathrm{C}(2)$ & $1.740(3)$ & $\mathrm{N}(2)-\mathrm{H}(2 \mathrm{~A})$ & 0.86 \\
$\mathrm{~N}(3)-\mathrm{C}(1)$ & $1.335(4)$ & $\mathrm{C}(2)-\mathrm{C}(3)$ & $1.357(5)$ \\
$\mathrm{N}(3)-\mathrm{C}(5)$ & $1.348(5)$ & $\mathrm{C}(4)-\mathrm{C}(5)$ & $1.369(6)$ \\
$\mathrm{N}(1)-\mathrm{N}(2)$ & $1.403(4)$ & $\mathrm{C}(4)-\mathrm{C}(3)$ & $1.389(6)$ \\
$\mathrm{N}(1)-\mathrm{H}(1 \mathrm{~A})$ & 0.86 & $\mathrm{C}(4)-\mathrm{H}(4 \mathrm{~A})$ & 0.93 \\
$\mathrm{~N}(1)-\mathrm{H}(1 \mathrm{~B})$ & 0.86 & $\mathrm{C}(5)-\mathrm{H}(5 \mathrm{~A})$ & 0.93 \\
$\mathrm{C}(1)-\mathrm{N}(2)$ & $1.365(4)$ & $\mathrm{C}(3)-\mathrm{H}(3 \mathrm{~A})$ & 0.93 \\
$\mathrm{C}(1)-\mathrm{C}(2)$ & $1.412(4)$ & - & - \\
\hline
\end{tabular}

TABLE-3

SELECTED BOND ANGLES $\left[^{\circ}\right]$ FOR THE TITLE COMPOUND

\begin{tabular}{cccc}
\hline $\begin{array}{c}\text { Bond } \\
\text { angles }\end{array}$ & $\begin{array}{c}\text { X-Ray } \\
\text { crystal }\end{array}$ & $\begin{array}{c}\text { Bond } \\
\text { angles }\end{array}$ & $\begin{array}{c}\text { X-Ray } \\
\text { crystal }\end{array}$ \\
\hline $\mathrm{C}(1)-\mathrm{N}(3)-\mathrm{C}(5)$ & $118.1(3)$ & $\mathrm{C}(3)-\mathrm{C}(2)-\mathrm{Cl}(1)$ & $120.9(3)$ \\
$\mathrm{N}(2)-\mathrm{N}(1)-\mathrm{H}(1 \mathrm{~A})$ & 120 & $\mathrm{C}(1)-\mathrm{C}(2)-\mathrm{Cl}(1)$ & $118.6(2)$ \\
$\mathrm{N}(2)-\mathrm{N}(1)-\mathrm{H}(1 \mathrm{~B})$ & 120 & $\mathrm{C}(5)-\mathrm{C}(4)-\mathrm{C}(3)$ & $118.4(3)$ \\
$\mathrm{H}(1 \mathrm{~A})-\mathrm{N}(1)-\mathrm{H}(1 \mathrm{~B})$ & 120 & $\mathrm{C}(5)-\mathrm{C}(4)-\mathrm{H}(4 \mathrm{~A})$ & 120.8 \\
$\mathrm{~N}(3)-\mathrm{C}(1)-\mathrm{N}(2)$ & $118.4(3)$ & $\mathrm{C}(3)-\mathrm{C}(4)-\mathrm{H}(4 \mathrm{~A})$ & 120.8 \\
$\mathrm{~N}(3)-\mathrm{C}(1)-\mathrm{C}(2)$ & $120.7(3)$ & $\mathrm{N}(3)-\mathrm{C}(5)-\mathrm{C}(4)$ & $123.8(3)$ \\
$\mathrm{N}(2)-\mathrm{C}(1)-\mathrm{C}(2)$ & $121.0(3)$ & $\mathrm{N}(3)-\mathrm{C}(5)-\mathrm{H}(5 \mathrm{~A})$ & 118.1 \\
$\mathrm{C}(1)-\mathrm{N}(2)-\mathrm{N}(1)$ & $121.5(3)$ & $\mathrm{C}(4)-\mathrm{C}(5)-\mathrm{H}(5 \mathrm{~A})$ & 118.1 \\
$\mathrm{C}(1)-\mathrm{N}(2)-\mathrm{H}(2 \mathrm{~A})$ & 119.3 & $\mathrm{C}(2)-\mathrm{C}(3)-\mathrm{C}(4)$ & $118.6(3)$ \\
$\mathrm{N}(1)-\mathrm{N}(2)-\mathrm{H}(2 \mathrm{~A})$ & 119.3 & $\mathrm{C}(2)-\mathrm{C}(3)-\mathrm{H}(3 \mathrm{~A})$ & 120.7 \\
$\mathrm{C}(3)-\mathrm{C}(2)-\mathrm{C}(1)$ & $120.5(3)$ & $\mathrm{C}(4)-\mathrm{C}(3)-\mathrm{H}(3 \mathrm{~A})$ & 120.7 \\
\hline
\end{tabular}

TABLE-4

SELECTED BOND ANGLES $\left[^{\circ}\right]$ TORSIONAL ANGELS $\left({ }^{\circ}\right)$ FOR THE TITLE COMPOUND

$\begin{array}{cc}\text { Bond angles } & \text { X-Ray crystal } \\ \mathrm{C}(5)-\mathrm{N}(3)-\mathrm{C}(1)-\mathrm{N}(2) & -177.6(3) \\ \mathrm{C}(5)-\mathrm{N}(3)-\mathrm{C}(1)-\mathrm{C}(2) & 0.4(5) \\ \mathrm{N}(3)-\mathrm{C}(1)-\mathrm{N}(2)-\mathrm{N}(1) & -9.2(5) \\ \mathrm{C}(2)-\mathrm{C}(1)-\mathrm{N}(2)-\mathrm{N}(1) & 172.8(3) \\ \mathrm{N}(3)-\mathrm{C}(1)-\mathrm{C}(2)-\mathrm{C}(3) & -0.3(5) \\ \mathrm{N}(2)-\mathrm{C}(1)-\mathrm{C}(2)-\mathrm{C}(3) & 177.7(3) \\ \mathrm{N}(3)-\mathrm{C}(1)-\mathrm{C}(2)-\mathrm{Cl}(1) & 178.4(3) \\ \mathrm{N}(2)-\mathrm{C}(1)-\mathrm{C}(2)-\mathrm{Cl}(1) & -3.6(5) \\ \mathrm{C}(1)-\mathrm{N}(3)-\mathrm{C}(5)-\mathrm{C}(4) & -0.1(5) \\ \mathrm{C}(3)-\mathrm{C}(4)-\mathrm{C}(5)-\mathrm{N}(3) & -0.3(6) \\ \mathrm{C}(1)-\mathrm{C}(2)-\mathrm{C}(3)-\mathrm{C}(4) & -0.2(6) \\ \mathrm{Cl}(1)-\mathrm{C}(2)-\mathrm{C}(3)-\mathrm{C}(4) & -178.8(3) \\ \mathrm{C}(5)-\mathrm{C}(4)-\mathrm{C}(3)-\mathrm{C}(2) & 0.5(6) \\ \end{array}$

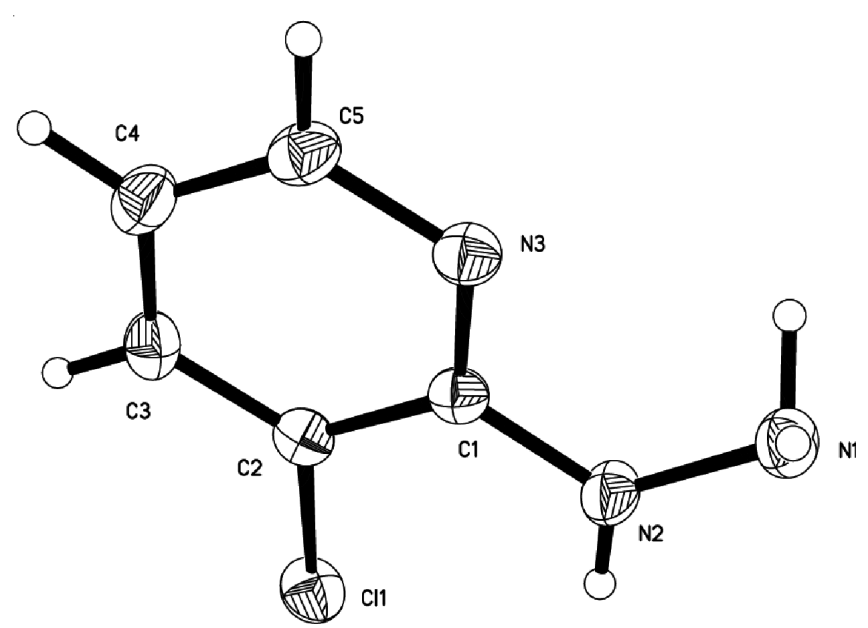

Fig. 1. Molecular structure of 3-chloro-2-hydrazinylpyridine 


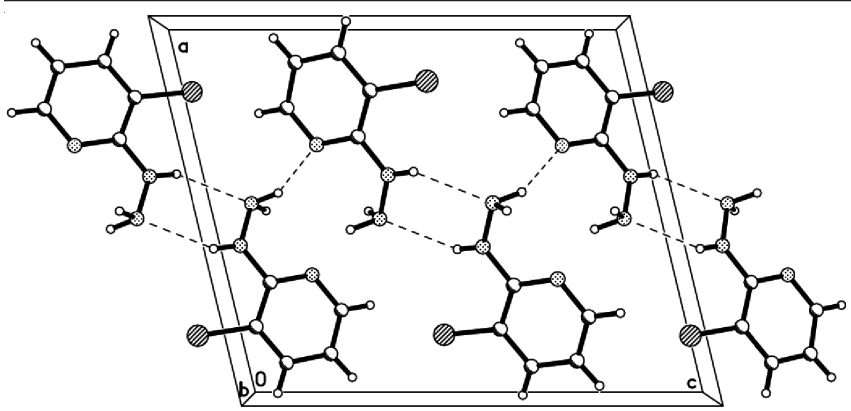

Fig. 2. The two-dimensional network of hydrogen bonds (dashed lines)

and N3-C1 are longer than the general $\mathrm{C}=\mathrm{N}$ double bond length of $1.27 \AA$. The N1-N2 bond lengths is 1.403(4) $\AA$. The bond angles of pyridine ring vary from $118.4(3)$ to $121.5(3)^{\circ}$ with the average of $120^{\circ}$.

The title compound has an extensive network of hydrogen bonding involving the two acceptor atoms $\mathrm{N}$. In the ac plane, they are linked together by $\mathrm{N}(1)-\mathrm{H}(1 \mathrm{C}) \cdots \mathrm{N}(2) \# 2, \mathrm{~N}(1)-$ $\mathrm{H}(1 \mathrm{~B}) \cdots \mathrm{N}(3) \# 2$ hydrogen bonds. This hydrogen-bonding sequence is repeated to form a ring. The ring has two $\mathrm{N}$ atoms at the vertices, leading to a hydrogen-bond network defining cyclic motifs denoted $\mathrm{R}_{2}{ }^{2}(6)$. The vertices are shared with neighbouring decagon to form an infinite two-dimensional network of hydrogen bonds in the ac plane.

Bioassay of fungicidal activities: Fungicidal activity of title compounds against Gibberella zeae (Schwein.) Petch., Alternaria solani (Ellis et Martin) Jones et Grout., Cercospora arachidicola, Botryosphaeria berengeriana f.sp. piricola (Nose) koganezawa et Sakuma, Fusarium oxysporum f.sp. cucumerinum, were determined according the reference. At the dose of $50 \mu \mathrm{g} / \mathrm{mL}$, the title compounds display moderate fungicidal activity against Gibberella zeae (Schwein.) Petch. (32\%), Alternaria solani (Ellis et Martin) Jones et Grout. (44\%), Cercospora arachidicola (18\%), Botryosphaeria berengeriana f.sp. piricola (Nose) koganezawa et Sakuma (51\%), Fusarium oxysporum f.sp. cucumerinum (21\%), respectively.

\section{REFERENCES}

1. (a). X.H. Liu, C.X. Tan and J.Q. Weng, Asian J. Chem., 23, 4064 (2011); (b). X.H. Liu, C.X. Tan and J.Q. Weng, Phosphorus Sulfur Silicon Rel. Elem., 186, 552 (2011); (c). X.H. Liu, J.Q. Weng, C.X. Tan, L. Pan,
B.L. Wang and Z.M. Li, Asian J. Chem., 23, 4031 (2011); (d). C.X. Tan, Y.X. Shi, J.Q. Weng, X. H. Liu, B.J. Li and W.G. Zhao, Lett. Drug Des. Discov., 9, 431 (2012); (e). J.Y. Tong, Y.X. Shi, X.H. Liu, N.B. Sun and B.J. Li, Chin. J. Org. Chem., 32, 2373 (2012); (f). X.H. Liu, W.G. Zhao, B.L. Wang and Z.M. Li, Res. Chem. Intermed., 38, 1999 (2012); (g). N.N. Su, Y. Li, S.J. Yu X. Zhang, X.H. Liu and W.G. Zhao, Res. Chem. Intermed., 39, 759(2012); (h. X.H.Liu, L. Pan, Y. Ma, J.Q. Weng, C.X. Tan, Y.H. Li, Y.X. Shi, B.J. Li, Z.M. Li and Y.G. Zhang, Chem. Biol. Drug Des., 78, 689 (2011); (i). X.H. Liu, J.Q. Weng, B.L. Wang, Y.H. Li, C.X. Tan and Z.M. Li, Res. Chem. Intermed., DOI: 10.1007/s11164-013-1113-4 (2013); (j) J.Y. Tong, N.B. Sun and H.K. Wu, J. Chem. Soc. Pak., 34, 1300 (2012); (k). N.B. Sun, J.Z. Jin, C. Lei and W. Ke, Asian J. Chem., 25, 4067(2013).

2. (a) Y.L. Xue, Y.G. Zhang and X.H. Liu, Asian J. Chem., 24, 3016 (2012); (b) P.Q. Chen, C.X. Tan, J.Q. Weng and X.H. Liu, Asian J. Chem., 24, 2808 (2012); (c) Y.L. Xue, Y.G. Zhang and X.H. Liu, Asian J. Chem., 24, 1571 (2012); (d) Y.L. Xue, Y.G. Zhang and X.H. Liu, Asian J. Chem., 24, 5087 (2012); (e) X.F. Liu and X.H. Liu, Acta Cryst., E67, o202 (2011); (f) X.H. Liu, L. Pan, C.X. Tan, J.Q. Weng, B.L. Wang and Z.M. Li, Pestic. Biochem. Physiol., 101, 143 (2011); (g) X.H. Liu, J.Q. Weng, C.X. Tan and H.J. Liu, Acta Cryst., E68, o493 (2012); (h). H.J. Liu, J.Q. Weng, C.X. Tan and X.H. Liu, Acta Cryst., E67, o1940 (2011); (i). X.H. Liu, C.X. Tan and J.Q. Weng, Phosphorus Sulfur Silicon Rel. Elem., 186, 558 (2011); (j) C.X. Tan, J.Q. Weng, Z.X. Liu, X.H. Liu and W.G. Zhao, Phosphorus Sulfur Silicon Rel. Elem., 187, 990 (2012); (k) J.Q. Weng, L. Wang and X.H. Liu, J. Chem. Soc. Pak., 34, 1248 (2012); (1) X.H. Liu, L. Pan, J.Q. Weng, C.X. Tan, Y.H. Li, B.L. Wang and Z.M. Li, Mol. Divers., 16, 251 (2012); (m) R.Wu, C. Zhu, X.J. Du, L.X. Xiong, S.J. Yu, X.H. Liu, Z.M. Li and W.G. Zhao, Chem. Cent. J., 6, 99 (2012); (n) X.H. Liu, J.Q. Weng and C.X. Tan, J. Chem., Article ID 306361 (2013); (o) X.F. Liu, X.W. Xiao and X.H. Liu, Chin. J. Struct. Chem., 30, 1437 (2011).

3. A.A. Salarian, A. Asadi-Eskandar, A. Sakhteman and K. Abdi, Asian J. Chem., 25, 1275 (2013).

4. (a) S.V. Rao, D. Komali, S.K. Ameer, S.D.V.V.S.S. Raju and K.N. Reddy, Asian J. Chem., 24, 3203 (2012); (b) S. Karabocek, E. Senses, N. Karabocek and S.A. Karaoglu, Asian J. Chem., 24, 188 (2012).

5. M.E.B. Rao and V.G. Rajurkar, Asian J. Chem., 23, 2648 (2011).

6. (a) B. Kumar, K.K. Prasad and S.K. Srivastava, Asian J. Chem., 23, 1680 (2011); (b) S. Benhammadi, A.A. Othman, A. Derdour and A. Mami, Asian J. Chem., 22, 5535 (2010); (c) V. Judge, B. Narasimhan, M. Ahuja, D. Sriram and P. Yogeeswari, Lett. Drug Des. Discov., 8, 792 (2011).

7. F.A. Bassyouni, H.A. Tawfik, A.M. Soliman and M.A. Rehim, Res. Chem. Intermed., 38, 1291(2012).

8. E.M. El-Telbani, S.M. Ghanem, F.A. El-Essawy and A.F. El-Sayed, Lett. Drug Des. Discov., 8, 822 (2011).

9. Y.B. Wang, Haiquan, W.L. Huang, H.B. Zhang and J.P. Zhou, Lett. Drug Des. Discov., 8, 500 (2011). 